\title{
Mathematical Model Development on the Deformation Behaviour of Symmetric Hexagonal of Various Angles and Square Tubes under Lateral Loading
}

\author{
M. Ghazali Kamardan', A. M. Ahmad Zaidi', ${ }^{2,3}$ Mohd Zaid Othman², M. Noh Dalimin ${ }^{1}$, \\ Suresh Thanakodi ${ }^{2} \&$ AK Mahamad ${ }^{4}$ \\ ${ }^{1}$ Faculty of Science, Technology and Human Development, Universiti Tun Hussein Onn Malaysia, Johor, \\ Malaysia \\ ${ }^{2}$ Faculty of Engineering, National Defence University of Malaysia, Kuala Lumpur, Malaysia \\ ${ }^{3}$ International College of Automotive, Pahang, Malaysia \\ ${ }^{4}$ Faculty of Electrical and Electronic Engineering, Universiti Tun Hussein Onn Malaysia, Johor, Malaysia \\ Correspondence: M. Ghazali Kamardan, Faculty of Science, Technology and Human Development, Universiti \\ Tun Hussein Onn Malaysia, Parit Raja, 86400 Batu Pahat, Johor, Malaysia. Tel: 60-167-157-357. E-mail: \\ mghazali@uthm.edu.my
}

Received: October 21, 2013 Accepted: December 8, 2013 Online Published: December 12, 2013

doi:10.5539/mas.v8n1p1

URL: http://dx.doi.org/10.5539/mas.v8n1p1

\begin{abstract}
The purpose of this research is to develop a mathematical model of the collapse behaviour of symmetric hexagonal tubes. For that, a finite element analysis procedure was conducted using ABAQUS software to determine the lateral collapse behaviour of symmetric hexagonal of angles, $\theta=30^{\circ}, 45^{\circ}$ and $60^{\circ}$ and square tubes to compare the results with the cylindrical tube. Then, a new predictive mathematical model of the lateral collapse behaviour for the generalized symmetrical geometric tubes is developed based on rigid, perfectly plastic model and the energy balance method. The newly mathematical model was validated with the simulation method results. It was discovered that symmetric hexagonal and square tubes performed different deformation behaviour than the cylindrical tube. Square and symmetric hexagonal with $\theta=15^{\circ}$ tubes performed Type II deformation behaviour. Symmetric hexagonal tubes with $\theta=30^{\circ}, 45^{\circ}$ and $60^{\circ}$ performed Type I with the perfectly plastic collapse behaviour whereas cylindrical tube performed Type I with strain hardening deformation behaviour. The mathematical prediction model had managed to model the deformation behaviour of symmetric hexagonal tubes with $\theta=30^{\circ}, 45^{\circ}$ and $60^{\circ}$ but failed to model the square and symmetric hexagonal with $\theta=15^{\circ}$ tubes because it was the perfectly plastic model which suitable for Type I with perfectly plastic deformation behaviour.
\end{abstract}

Keywords: Lateral collapse, symmetric hexagonal tubes, square tube, mathematical model, energy absorption

\section{Introduction}

The crashworthiness or the response quality of a vehicle during collision or impact has become one of the important engineering studies in the designing of vehicles. This study is important in order to improve on the crashworthy properties of a vehicle so that the fatalities rate on the occupants involved in the crash could be reduced (Johnson, 1990). When a collision occur, the energy absorption system should react by absorbing and dissipating the kinetic energy into an irreversible or inelastic energy such as plastic deformation, viscous energy and friction or fracture energy (Olabi, Morris, \& Hashmi, 2007). One of the popular energy absorption systems is the lateral compression of cylindrical tube.

Several experimental and analytical studies which basically focused on the cylindrical tube had been performed with regards to the lateral collapse of tubes compressed between two flat rigid plates. The first mathematical model was developed based on rigid perfectly plastic material model where the load-deformation prediction was based on the energy balance method and it took into account the geometrical components of stiffening phenomenon (De Runtz \& Hodge, 1963). The model included four stationary plastic hinges where the plastic deformation occurred to replicate the experimental quasi-static lateral collapse of mild steel cylindrical tube which was compressed between two flat rigid plates. Another separate mathematical model assumed that there were six plastic hinges 
around the perimeter of the tube and the ring straightened at the moving contact point (Burton \& Craig, 1963). However both mathematical models (Burton \& Craig, 1963; De Runtz \& Hodge, 1963) did not take into account the material strain hardening hence the actual results were under estimated. Those mathematical models were then improved by introducing a rigid linear strain hardening material model (Redwood, 1964). Lastly, the mathematical model was improved further by relating the contribution of strain hardening over a variable length of plastic hinges (Reid \& Reddy, 1978). This vastly improved mathematical model was to be the most accurate with the experimental results and still being applied until now. However, the focus of all these mathematical models was on cylindrical tube.

Several studies on the impact loading have focussed on the geometrical tube's shapes such as hexagon and octagon in the last few years to find the alternative of cylindrical and square tubes (Abdewi, Sulaiman, Hamouda, \& Mahdi, 2008; Radzi et al., 2013; Song, Chen, \& Lu, 2012; X. Zhang \& Huh, 2009; Z. Zhang, Liu, \& Tang, 2010). These researches were conducted mainly to increase the plastic deformation zones and hence improved the energy absorption efficiency. This was to reduce the initial peak and the subsequent fluctuations in the load-deflection curve and improved the uniformity of the load-displacement curve which showed a great potential of geometrical tube's shape. However, the studies of these geometrical shapes effects are still new and more studies need to be conducted to improve the results obtained and to deliver more convincing results.

During the compression process, the cylindrical tube was deformed by undergoing three major phases which resulted in three stages in the force-deformation curve. The first stage was the linear increasing force, followed by a flat force, an increasing force or a curve and lastly an unbounded increasing force. The first stage was for the elastic collapse, the second stage was for the plastic collapse and the last stage was for the densification of the cylindrical tube (Gibson \& Ashby, 1997). During the plastic collapse, three types of behaviour can take place which are the strain hardening, strain softening and perfectly-plastic (Li, Magkiriadis, \& Harrigan, 2006). Strain hardening is the case where the stress will continue to increase as the deformation continues after the yield stress, $\sigma_{y}$. Strain softening is the case where the stress will reduce as the deformation continues after the yield stress. Perfectly-plastic is the case where the stress will remain at the yield stress, $\sigma_{y}$ as the deformation continues. Figure 1(a)-(c), the stress - strain relationship illustrates these behaviours. In these figures, $\sigma$ represents the stress and $\varepsilon$ represents the strain. There were two type force-displacement curves under the compression loading, named as Type-I and Type-II (Calladine \& English, 1984). Type I executes flat force whereas Type-II performs an initial peak force and then followed by an immediately reduced force. These conditions are illustrated in Figure 2.

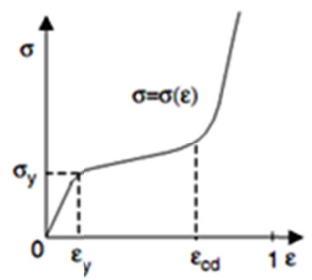

(a)

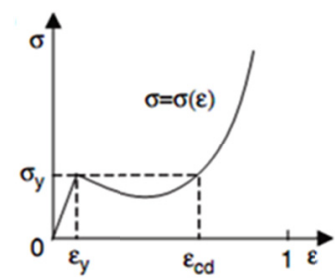

(b)

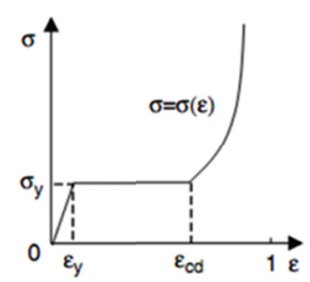

(c)

Figure 1. Collapse behaviour of tube under lateral compression (a) Strain-hardening behaviour, (b) Strain-softening behaviour and (c) Perfectly-plastic behaviour (Li et al., 2006)

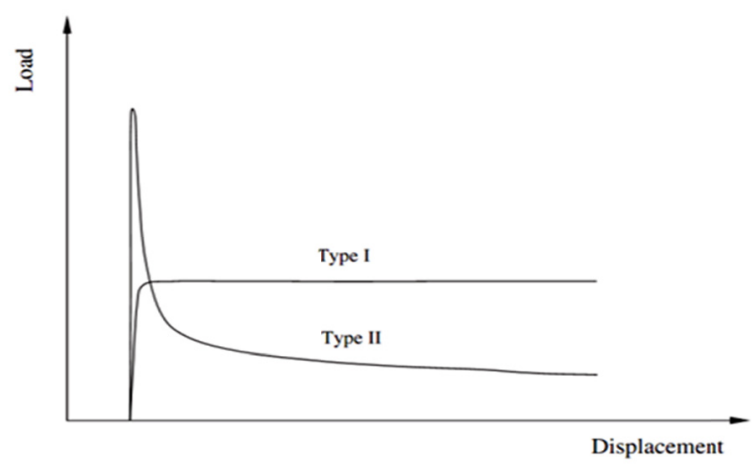

Figure 2. The force vs displacement relationship of Type-I and Type-II deformation behaviour pattern (Calladine \& English, 1984) 
Based on the previous works, an attempt has been performed to develop a mathematical model to predict the collapse behaviour of symmetric hexagonal and square tubes. This mathematical model is discussed in section two. The mathematical works were then being validated with the finite element analysis (FEA) simulation results. The simulations were conducted by using a finite element analysis software i.e. ABAQUS to study the collapse behaviour of symmetric hexagonal tubes. The simulation procedure is discussed in section three. In section four, the results of the deformation behaviour analyses and the mathematical model validation are discussed. Then, the discussion of the results is presented in section five. Lastly, in section six a conclusion of the study is included.

\section{Mathematical Model}

\subsection{Hexagon}

Consider the equilibrium of a segment in a symmetric hollow hexagonal shape as shown in Figure 3 . Let $H, L, R$ and $\theta$ as the total height, total length, the length of an oblique side and the angle between vertical axis and the oblique side respectively.

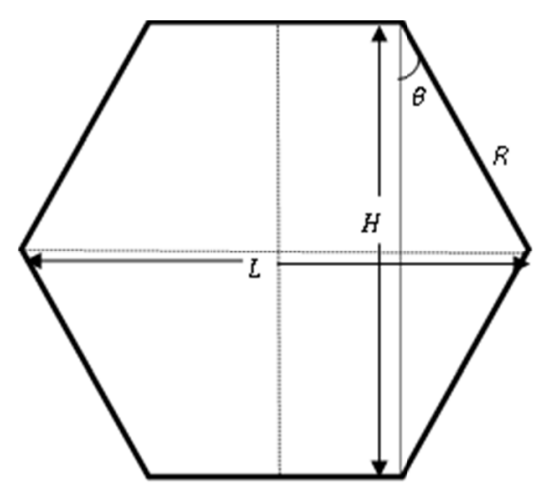

Figure 3. Symmetric hexagonal shape

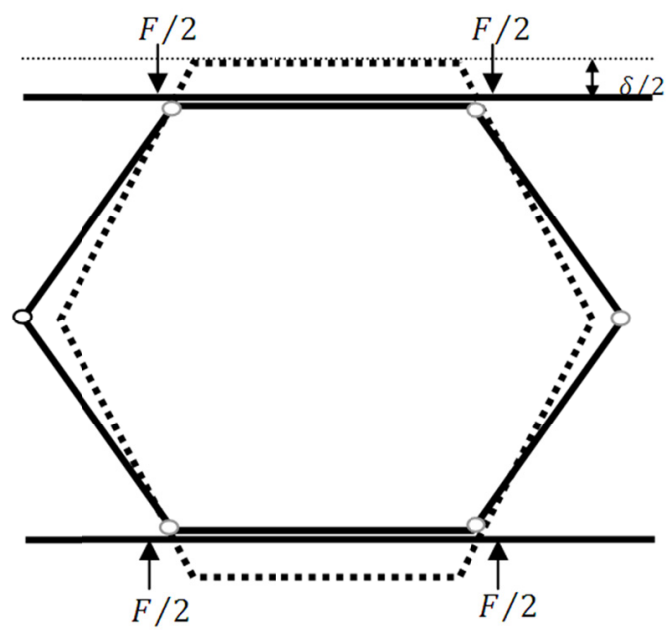

Figure 4. Symmetric hollow hexagon shape compressed between two rigid plates

The symmetric hollow hexagonal shape was placed in between two flat rigid plates as shown Figure 4. The top flat rigid plate was positioned on top of the shape that acted as the compressor which provided the downwards axial loading while the bottom plate stayed stationary and acted as the fixed base. It was expected that there were six stationary plastic hinges for symmetric hexagonal tube at all the vertices of the hexagon which forced all of the four oblique sides to collapse until the wall of top half of the hexagon contacted with the wall of bottom half of the hexagon and the symmetric hexagonal tube will become flat. 


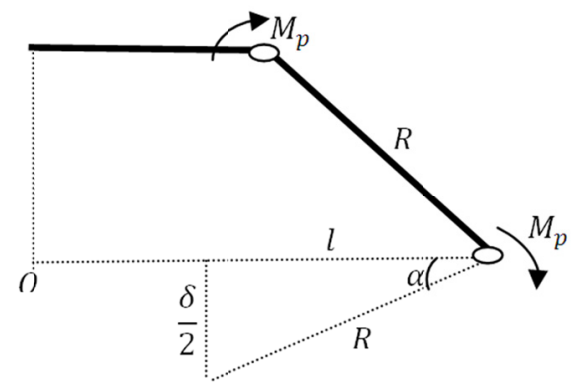

Figure 5. The point of hinge of the symmetric hollow hexagon shapes during the lateral collapse

Since the symmetric hexagonal tube was compressed between the two rigid plates, the forces and moments which acted on the top half and bottom half of the tube were equal i.e. $F / 2$. As the hexagonal tube was symmetric, the deformations at all four quadrants were expected to be similar. Let's just consider the first quadrant (refer Figure 5). At the equilibrium, the forces and moments worked along the same horizontal line in the middle of the hexagon of length $l$. At the beginning $l=R \cos \alpha$, where $\alpha=0$. Thus $l=R$.

For an angle of segment rotation $\alpha$, the compression was

$$
\begin{aligned}
& R=\frac{H}{2 \cos \theta} \\
& \frac{\delta}{2}=R \sin \alpha \\
& l=R \cos \alpha
\end{aligned}
$$

From the energy balance: $E_{\text {in }} \equiv P * \Delta *=D=\sum_{i=1}^{n} M_{p}\left|\theta_{i}\right|$

The elastic collapse of the tube was very small when compared to the plastic collapse and thus for simplification, the elastic deformation process can be discarded from the development of the mathematical analysis ( $\mathrm{Lu} \& \mathrm{Yu}$, 2003). Thus, a rigid, perfectly plastic model was used as the collapse model. Based on the energy balance method, the force $F / 2$ was equal to $2 M_{p} / l$, where $M_{p}=Y b h^{2} / 4$ was the fully plastic bending moment of the ring with $b$ was the width of the ring or tube and $h$ the thickness of the ring or tube. Hence

$$
\begin{aligned}
\frac{F}{2} & =\frac{2 M_{p}}{l} \\
\frac{2 M_{p}}{F} & =\frac{R \cos \alpha}{2} \\
F & =\frac{4 M_{p}}{R \cos \alpha}
\end{aligned}
$$

The initial collapse load was therefore

$$
\begin{gathered}
F_{0}=\frac{Y b h^{2}}{R} \\
\frac{F}{F_{0}}=\frac{1}{\left[1-\left(\frac{\delta}{2 R}\right)^{2}\right]^{1 / 2}}
\end{gathered}
$$

Substituted (1) and (7) into (8). Then, the deformation could not exceed the total length of the shape, $H=$ $2 R \cos \theta$. After this point, densification occurred and force, $F$ will be unbounded. Then, the model of the lateral collapse of symmetric hexagonal tube was defined as follow:

$$
\begin{aligned}
F & =\left\{\begin{array}{ccc}
\frac{Y b h^{2}}{R\left[1-\left(\frac{\delta}{2 R}\right)^{2}\right]^{\frac{1}{2}}} & \text { for } & 0<\delta \leq 2 R \cos \theta ; \\
\infty & \text { for } & \delta>2 R \cos \theta .
\end{array}\right. \\
\text { Or } \quad F & =\left\{\begin{array}{ccc}
\frac{2 Y b h^{2} \cos \theta}{H\left[1-\left(\frac{\delta}{H} \cos \theta\right)^{2}\right]^{\frac{1}{2}}} & \text { for } & 0<\delta \leq H ; \\
\infty & \text { for } & \delta>H .
\end{array}\right.
\end{aligned}
$$




\subsection{Square}

Figure 6 is the diagram of a square shape. By assuming that the equilibrium of a segment in a hollow square was just like the equilibrium of a segment of symmetric hollow hexagon with $H=L, \theta=0$ and $R=H / 2$. Similar to hollow hexagon, there were six stationary plastic hinges for hollow square which were at the four vertices and at middle of the vertical sides of the shape that will make the vertical sides deformed and the horizontal will remain unchanged.

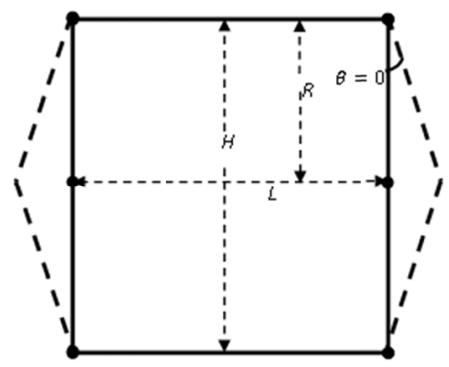

Figure 6. Square shape

Based on the hexagon shape, for comparison, by letting $\theta=0$, the hexagon shape would form a square shape. From (2), since $\theta=0$, then $R=H / 2$. Thus the model of the lateral collapse of symmetric square tube was given as

$$
\begin{aligned}
& F=\left\{\begin{array}{llr}
\frac{Y b h^{2}}{R\left[1-\left(\frac{\delta}{2 R}\right)^{2}\right]^{\frac{1}{2}}} & \text { for } & 0<\delta \leq 2 R ; \\
\infty & \text { for } & \delta>2 R .
\end{array}\right. \\
& \text { Or } F=\left\{\begin{array}{ccc}
\frac{2 Y b h^{2}}{H\left[1-\left(\frac{\delta}{H}\right)^{2}\right]^{\frac{1}{2}}} & \text { for } & 0<\delta \leq H ; \\
\infty & \text { for } & \delta>H .
\end{array}\right.
\end{aligned}
$$

\section{Simulation Procedures}

The simulation procedure was performed by using a finite element analysis software i.e. ABAQUS (Hibbitt, Karlsson, \& Sorensen, 2006). The lateral collapse of various symmetric hexagonal and square tubes of different angles compressed between two flat rigid plates was simulated using quasi static analyses. Three dimensional (3D) model was developed by using the Arbitrary Lagrange Euler (ALE) solver by setting the analysis according to the "Static-General Implicit" analysis procedure. The shape of the symmetric hexagonal tubes was modelled by using a 3D modelling space, deformable behaviour with a solid extrude base feature. The rigid plates were modelled by using a 3D modelling space and the 'discrete rigid' type behaviour with a shell extrude base feature. Both flat rigid plates were assigned with $100 \mathrm{R} 3 \mathrm{D} 4$ or quadrilateral rigid elements. All of the geometric symmetric tubes were meshed by using 15000 to 30000 'C3D8R' type elements or 8 node brick elements. Mesh discretisation method was performed to get the optimum number of elements (Koslan et al., 2013). The material properties were set based on the stainless properties, i.e. Young's modulus, $\mathrm{E}=200 \mathrm{GPa}$, Poisson's ratio, $v=0.35$. yield strength, $\sigma_{\mathrm{Y}}=207 \mathrm{MPa}$ and ultimate strength, $\sigma_{\mathrm{U}}=517 \mathrm{MPa}$.

The top and bottom flat rigid plates were assumed to be rigid bodies where there were no deformations for both flat rigid plates during the compression. The simulations were performed by placing the object in between two flat rigid plates (see Figure 7). The bottom flat rigid plate was set to be fully fixed at its location while the top flat rigid plate which acts as the compressor was set as moving in negative y-axis (downward) direction so that the object can be compressed. The frictional effect between the flat rigid plates and the object was neglected. 


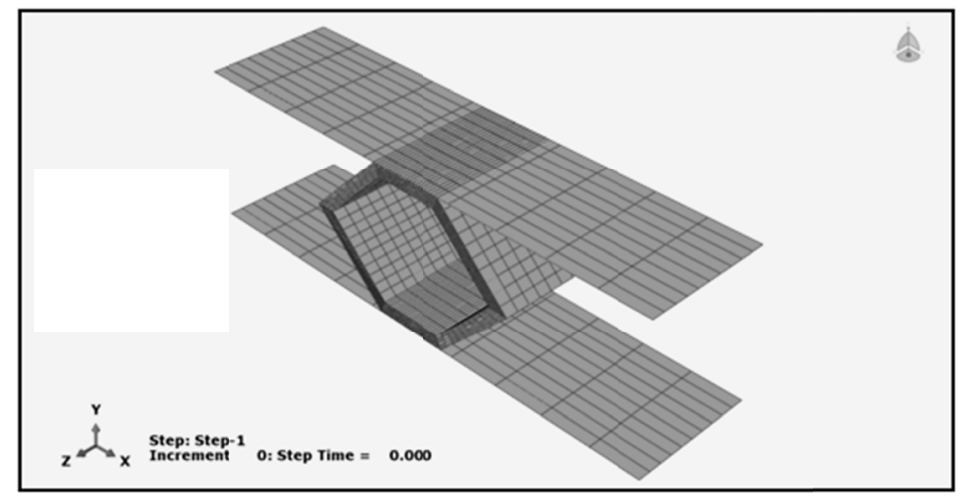

Figure 7. The diagram of symmetric hexagonal tube placed in between two flat rigid plates

\section{Results}

4.1 Comparison of the Deformation Behaviour among Cylindrical, Square and Various Symmetric Hexagonal Tubes Based on Simulation Method

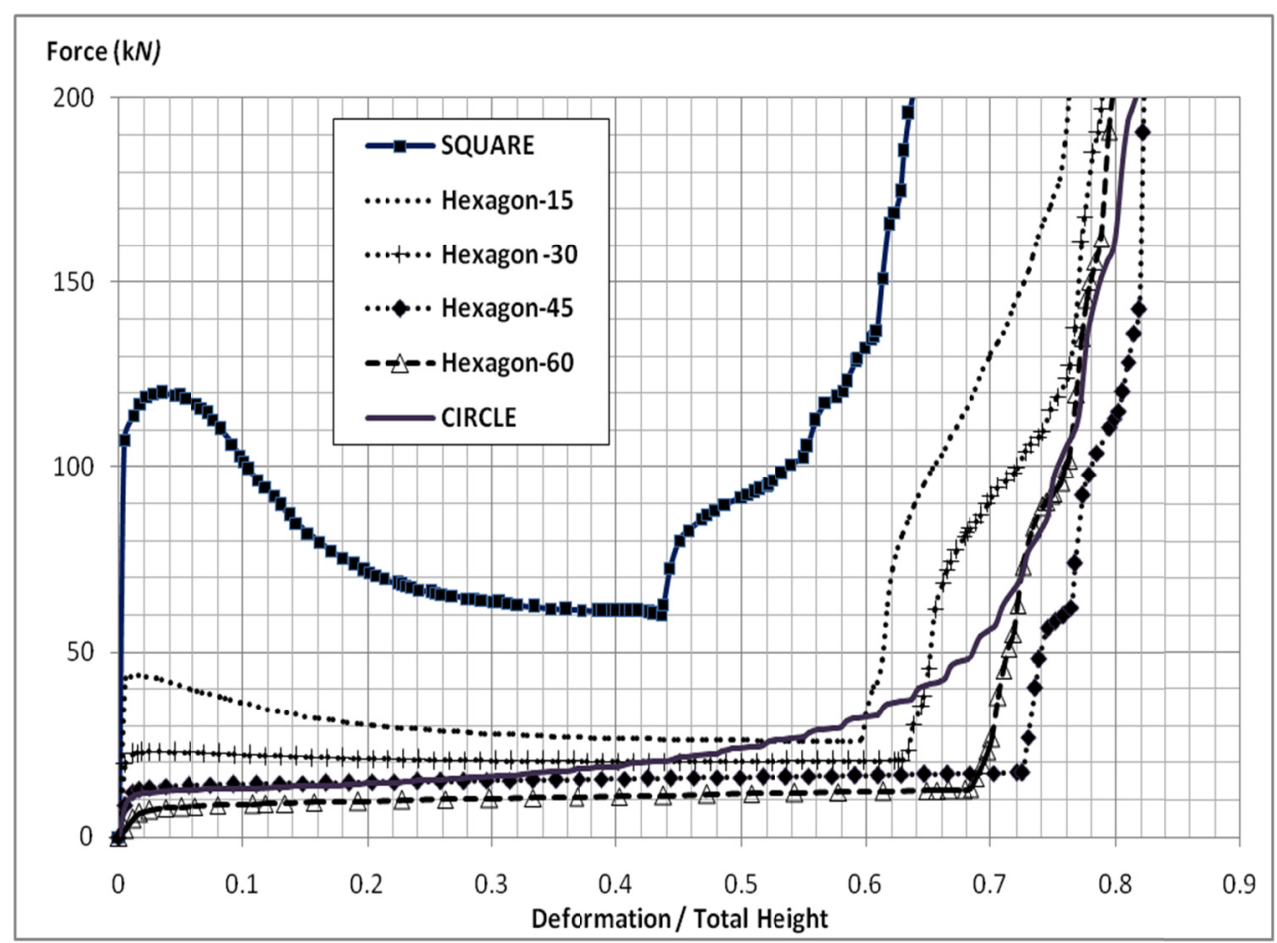

Figure 8. Force vs deformation/ total height relationship for cylindrical, square and symmetric hexagonal of various angles, $\theta=15^{\circ}, \mathbf{3 0}^{\circ}, \mathbf{4 5}^{\circ}$ and $60^{\circ}$ tubes

Symmetric hexagonal tubes with different angles, $\theta=15^{\circ}, 30^{\circ}, 45^{\circ}$ and $60^{\circ}$ (see Figure 3 ) which had been compressed laterally, between two flat rigid plates and their collapse behaviours were compared to the ones of square and circle tubes. Square tube could be assumed to have similar geometry as symmetric hexagonal tubes with $\theta=0^{\circ}$ (see Figure 6). These collapse behaviors were obtained from the observation of the force vs deformation relationship as shown in Figure 8. It can be observed that, the square and all the symmetric hexagonal tubes in this study had produced different deformation behaviours than cylindrical tube.

The cylindrical tube had an immediate increasing force, followed by a steadily increasing force and lastly, the force increased unboundedly. On the other hand, for square tube, there was as a high initial peak which then immediately reduced and lastly, the force then rose unboundedly. Symmetric hexagonal tube with $\theta=15^{\circ}$ also had an initial peak but the peak was shallow. The height of the peak was one third of the height of the initial 
peak of square tube. For the other symmetric hexagonal tubes, there was an immediately increasing force, followed by a flat or nearly flat force and ended with an unbounded rising force. The flat force of the symmetric hexagonal tubes became lower and longer when the value of $\theta$ was increased.

4.2 Validation of Mathematical Model Results with Simulation Results for Square and Various Symmetric Hexagonal Tubes

a)

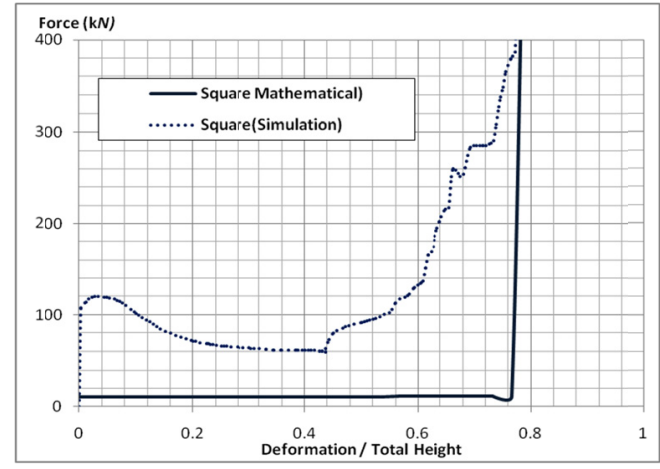

c)

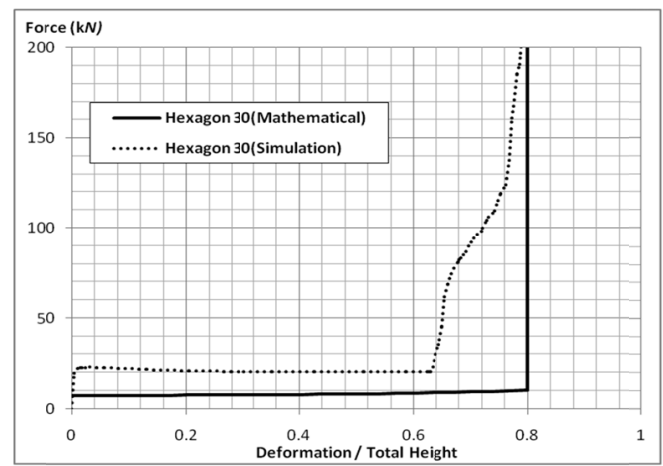

b)

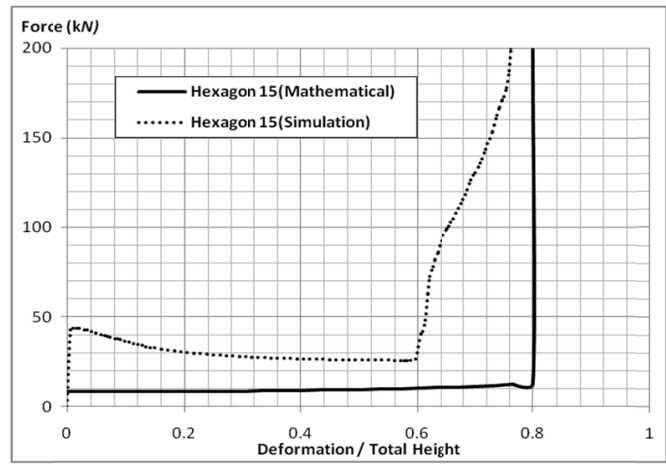

d)

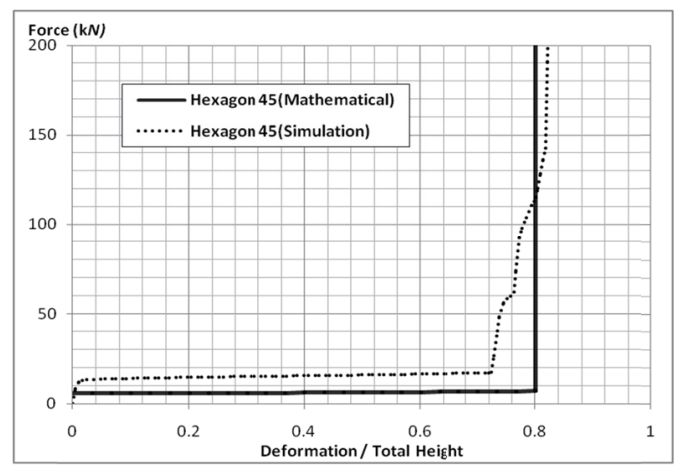

e)

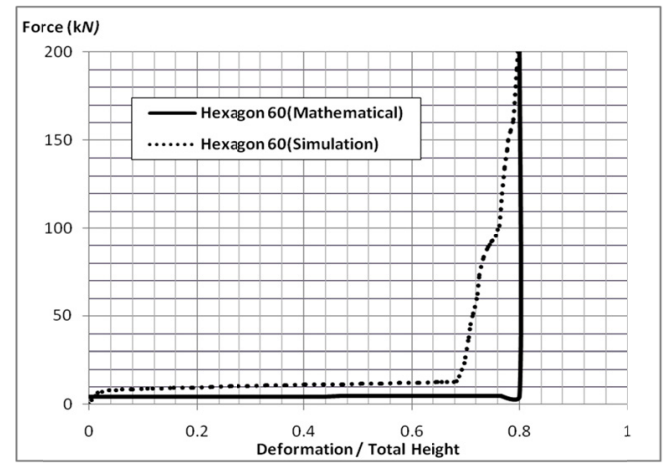

Figure 9. Comparing mathematical against simulation results of force vs deformation/total height relationship of square and symmetrical hexagonal of various angle, $\theta$ tubes: (a) square $\left(\theta=0^{\circ}\right),(\mathrm{b}) \theta=15^{\circ},(\mathrm{c}) \theta=30^{\circ}$, (d) $\theta=45^{\circ}$ and (e) $\theta=60^{\circ}$

The diagrams in Figure 9 (a) - (e) demonstrate the comparison of the force vs deformation/total height obtained from the simulation processes and the mathematical models for the square and symmetric hexagonal with angles of $\theta=15^{\circ}, 30^{\circ}, 45^{\circ}$ and $60^{\circ}$ tubes respectively. Figure 9 (a) shows that the mathematical model results did not well agree with the simulation results. The square tube in Figure 9 (a) had the highest gap between simulation results and mathematical model results. However, the gap between both results was reduced when the angle, $\theta$ was increased. The diagram in Figure 9 (e) shows the closest result between both methods when $\theta=60^{\circ}$. Figures 9 (a) and (b) show that for square and symmetric hexagonal with $\theta=15^{\circ}$ tubes under simulation methods, the deformation curves had an initial peak, followed by a reduced curve and ended with an unbounded increasing force. 
Figures 9 (c) - (e) show that the symmetric hexagonal with $\theta=30^{\circ}, 45^{\circ}$ and $60^{\circ}$ tubes had a flat or nearly flat force and ended with high rising force. Figures 9 (a) - (e) show that under the mathematical model methods, all the diagrams had a flat force and ended with high rising force.

\section{Discussion}

The comparison of force vs deformation/total height relationship under the simulation method for cylindrical, square and symmetric hexagonal of the various angles i.e. $\theta=15^{\circ}, 30^{\circ}, 45^{\circ}$ and $60^{\circ}$ tubes had shown that the square and symmetric hexagonal tubes was different than the cylindrical tube regarding the deformation behviour pattern at the plastic collapse zone. All the tube structures had an early and immediately increasing force which was the elastic collapse zone. Then, there was the plastic collapse zone where all of the three types of structures had shown the different behaviours. The cylindrical tube had a steadily increasing curve force, the square and symmetric hexagonal with $\theta=15^{\circ}$ tubes had an initial peak, and the rest of symmetric hexagonal tubes i.e. with $\theta=30^{\circ}, 45^{\circ}$ and $60^{\circ}$ tubes had a long flat or nearly flat force. For all of the tube structures in this study, the force ended with a high rising force which was the densification zone for all the tubes.

The initial peak phenomena for square and symmetric hexagonal with $\theta=15^{\circ}$ tubes was in accordance with the Type II deformation behaviour (Calladine \& English, 1984) since a high early force was needed against the elastic deformation of the vertical sides of square tube and the oblique sides with acute angle of symmetric hexagonal with $\theta=15^{\circ}$ tube before plastic deformation could take place. The cylindrical and symmetric hexagonal with $\theta=30^{\circ}, 45^{\circ}$ and $60^{\circ}$ tubes closely resembled the Type I deformation behaviour with the long flat or nearly flat or the steady increasing force (Calladine \& English, 1984) since no high force was needed to collapse their oblique sides. For this type of collapse behaviour, the early linear increasing force is the elastic deformation zone, the following long flat force or plateau is the plastic deformation zone and the last high rising force is the densification zone (Gibson \& Ashby, 1997).

The plastic collapse for the cylindrical tube was due to the four stationary hinges (De Runtz \& Hodge, 1963) or six hinges around the perimeter of the cylindrical tube (Burton \& Craig, 1963). Based on the mathematical model prediction and simulation performed, the plastic collapse for symmetric hexagonal tubes was due to the stationary plastic hinge at all the six vertices which made the four oblique sides at the left and right of the symmetric hexagonal tubes to collapse. The steadily increasing force of the cylindrical tube resembled the strain hardening deformation whereas the flat force of the symmetric hexagonal tubes resembled the perfectly plastic deformation behaviour ( $\mathrm{Li}$ et al., 2006).

For square and symmetric hexagonal tubes, the yield stress $\sigma_{\mathrm{Y}}$ was reduced when the angle $\theta$ was increased. For square tube, $\sigma_{\mathrm{Y}}=120 \mathrm{kN}$, for hexagon tubes, when $\theta=15^{\circ}, \sigma_{\mathrm{Y}}=40 \mathrm{kN}$, when $\theta=30^{\circ}, \sigma_{\mathrm{Y}}=20 \mathrm{kN}$, when $\theta=45^{\circ}, \sigma_{\mathrm{Y}}=10 \mathrm{kN}$ and when $\theta=60^{\circ}, \sigma_{\mathrm{Y}}=5 \mathrm{kN}$. This shows that the increased of $\theta$ has reduced the elastic deformation and hence reducing the possibility of injury and damage due to the effect of repetitive acceleration and deceleration under the elastic deformation zone (Johnson \& Reid, 1978).

In contrast, the long flat force or the stroke length became longer when the angle $\theta$ was increased. For square tube the percentage of stroke length to the tube's size was $44 \%$. The stroke length percentage for hexagon tubes was $60 \%$ when $\theta=15^{\circ}, 64 \%$ when $\theta=30^{\circ}$, and $72 \%$ when $\theta=45^{\circ}$, but slightly reduced to $68 \%$ when $\theta=60^{\circ}$. The longer stroke will produce a longer plastic displacement, increasing the rate of hindering after an impact and hence will reduce the possibility of severe injury and damage ( $\mathrm{Lu} \& \mathrm{Yu}, 2003$ ). Thus, this shows that the increased of $\theta$ has improved the energy-absorption mechanism of symmetric hexagonal tubes.

The validation of the mathematical model with the simulation method showed a big gap for square and symmetric hexagonal tubes with a small angle, $\theta$. The gap was reduced when the angle was increased and when $\theta=60^{\circ}$ the result was almost close. The gap was due to the yield stress, $\sigma_{\mathrm{Y}}$ for square and symmetric hexagonal tubes with small $\theta$ were very high. This was because the deformation behaviour for square tube and the symmetric hexagonal tube with small $\theta$ were in accordance with the Type II and the deformation behaviour gradually change to the Type I behaviour as the angle $\theta$ for symmetric hexagonal tube was increased. Since, the mathematical model was based on the rigid, perfectly plastic model which resembled Type I deformation behaviour, it showed contrasting results to the Type II deformation behaviour. However, the mathematical model had managed to show the general deformation pattern of the symmetric hexagonal tubes where there is an existence of a long flat force before densification occurred at around $80 \%$ of the collapse. 


\section{Conclusion}

The validation of the mathematical model with the simulation method has shown that for square and symmetric hexagonal tubes with $=15^{\circ}$, both methods did not have good agreement since these tubes had Type II pattern with an initial peak but the mathematic model was based on the rigid, perfectly plastic model which resembled Type I deformation behaviour without the initial peak. When the angle $\theta$ for symmetric hexagonal tubes was increased, the deformation behaviour of the hexagonal tubes had gradually changed to the Type I behaviour which showed the agreement of both methods was getting better. The symmetric hexagonal tubes with $\theta=60^{\circ}$ showed very close results between both methods.

For symmetric hexagonal tubes, the increased of the angle $\theta$ had reduced the yield stress $\sigma_{\mathrm{Y}}$ but increased the plastic deformation thus improved the energy-absorption system by reducing the possibility of damage and injury during the collision.

\section{References}

Abdewi, E. F., Sulaiman, S., Hamouda, A. M. S., \& Mahdi, E. (2008). Quasi-static axial and lateral crushing of radial corrugated composite tubes. Thin-Walled Structures, 46(3), 320-332. http://dx.doi.org/10.1016/j.tws.2007.07.018

Burton, R. H., \& Craig, J. M. (1963). An investigation into the energy absorbing properties of metal tubes loaded in the transverse direction. University of Bristol, United Kingdom.

Calladine, C. R., \& English, R. W. (1984). Strain-rate and inertia effects in the collapse of two types of energy-absorbing structure. International Journal of Mechanical Sciences, 26(11-12), 689-701. http://dx.doi.org/10.1016/0020-7403(84)90021-3

De Runtz, J. A., \& Hodge, P. G. (1963). Crushing of a tube between rigids plates. Journal of Applied Mechanics, 30, 391-395. http://dx.doi.org/10.1115/1.3636567

Gibson, L. J., \& Ashby, M. F. (1997). Cellular solids: structure and properties (pp. 175-183). Cambridge: Cambridge University Press.

Hibbitt, D., Karlsson, B., \& Sorensen, P. (2006). ABAQUS Analysis User's Manual. In ABAQUS Version 6.6. USA.

Johnson, W. (1990). The elements of crashworthiness: scope and actuality. In Proceeding Institution of Mechanical Engineers (Vol. 204, pp. 255-273).

Johnson, W., \& Reid, S. R. (1978). Metallic Energy Dissipating Systems. Applied Mechanics Reviews, 31, 277-288.

Koslan, F. S., Mujahid, A., Zaidi, A., Othman, M. Z., Abdullah, S., \& Thanakodi, S. (2013). The Effect of Mesh Sizing Toward Deformation Result in Computational Dynamic Simulation for Blast Loading Application. Modern Applied Science, 7(7), 23-28. http://dx.doi.org/10.5539/mas.v7n7p23

Li, Q. M., Magkiriadis, I., \& Harrigan, J. J. (2006). Compressive Strain at the Onset of Densification of Cellular Solids. Journal of Cellular Plastics, 42, 371-392. http://dx.doi.org/10.1177/0021955X06063519

Lu, G., \& Yu, T. (2003). Energy absorption of structures and materials. Cambridge, England: Woodhead Publishing Limited.

Olabi, A. G., Morris, E., \& Hashmi, M. S. J. (2007). Metallic tube type energy absorbers : A synopsis. Thin Walled Structures, 45, 706-726. http://dx.doi.org/10.1016/j.tws.2007.05.003

Radzi, A., Ghani, A., Kee, C. S., Othman, M. Z., Koslan, F. S., \& Mujahid, A. (2013). Impact Response of Multi-Grooved Square Column. Modern Applied Science, 7(11), 12-25. http://dx.doi.org/10.5539/mas.v7n11p12

Redwood, R. G. (1964). Discussion on the paper by De Runtz, J. A. and Hodge Jr. P. G. (1963). Journal of Applied Mechanics, 31, 357-358. http://dx.doi.org/10.1115/1.3629622

Reid, S. R., \& Reddy, T. Y. (1978). Effect of strain hardening on the lateral compression of tubes between rigid plates. Int J Solids Struct, 14, 213-225. http://dx.doi.org/10.1016/0020-7683(78)90026-4

Song, J., Chen, Y., \& Lu, G. (2012). Axial crushing of thin-walled structures with origami patterns. Thin Walled Structures, 54, 65-71. http://dx.doi.org/10.1016/j.tws.2012.02.007

Zhang, X., \& Huh, H. (2009). Energy absorption of longitudinally grooved square tubes under axial compression. Thin Walled Structures, 47(12), 1469-1477. http://dx.doi.org/10.1016/j.tws.2009.07.003 
Zhang, Z., Liu, S., \& Tang, Z. (2010). Crashworthiness investigation of kagome honeycomb sandwich cylindrical column under axial crushing loads. Thin Walled Structures, 48(1), 9-18. http://dx.doi.org/10.1016/j.tws.2009.08.002

\section{Copyrights}

Copyright for this article is retained by the author(s), with first publication rights granted to the journal.

This is an open-access article distributed under the terms and conditions of the Creative Commons Attribution license (http://creativecommons.org/licenses/by/3.0/). 